\title{
UCRL-CONF-208349
}

LAW RENCE LIVERMORE N A TIO N A L LABORATORY
Development of Burning Plasma and Advanced Scenarios in the DIII-D Tokamak

T. C. Luce

December 2, 2004

20th IAEA Fusion Energy Conference Vilamoura, Portugal

November 1, 2004 through November 6, 2004 
This document was prepared as an account of work sponsored by an agency of the United States Government. Neither the United States Government nor the University of California nor any of their employees, makes any warranty, express or implied, or assumes any legal liability or responsibility for the accuracy, completeness, or usefulness of any information, apparatus, product, or process disclosed, or represents that its use would not infringe privately owned rights. Reference herein to any specific commercial product, process, or service by trade name, trademark, manufacturer, or otherwise, does not necessarily constitute or imply its endorsement, recommendation, or favoring by the United States Government or the University of California. The views and opinions of authors expressed herein do not necessarily state or reflect those of the United States Government or the University of California, and shall not be used for advertising or product endorsement purposes. 


\title{
$20^{\text {th }}$ IAEA Fusion Energy Conference \\ Vilamoura, Portugal, 1 to 6 November 2004
}

IAEA-CN-116/OV/1-3

\section{DEVELOPMENT OF BURNING PLASMA AND ADVANCED SCENARIOS IN THE DIII-D TOKAMAK}

\author{
T.C. LUCE for the DIII-D TEAM
}

General Atomics

San Diego, California 92186-5608

United States of America

\begin{abstract}
This is a preprint of a paper intended for presentation at a scientific meeting. Because of the provisional nature of its content and since changes of substance or detail may have to be made before publication, the preprint is made available on the understanding that it will not be cited in the literature or in any way be reproduced in its present form. The views expressed and the statements made remain the responsibility of the named author(s); the views do not necessarily reflect those of the government of the designating Member State(s) or of the designating organization(s). In particular, neither the IAEA nor any other organization or body sponsoring this meeting can be held responsible for any material reproduced in this preprint.
\end{abstract}




\title{
Development of Burning Plasma and Advanced Scenarios in the DIII-D Tokamak
}

\author{
T.C. Luce for the DIII-D Team* \\ General Atomics, P.O. Box 85608, San Diego, California 92186-5608, USA \\ e-mail contact of main author: luce@fusion.gat.com
}

\begin{abstract}
Significant progress in the development of burning plasma scenarios, steady-state scenarios at high fusion performance, and basic tokamak physics has been made by the DIII-D Team. Discharges similar to the ITER baseline scenario have demonstrated normalized fusion performance nearly $50 \%$ higher than required for $Q=10$ in ITER, under stationary conditions. Discharges that extrapolate to $Q \sim 10$ for longer than one hour in ITER at reduced current have also been demonstrated in DIII-D under stationary conditions. Proof of high fusion performance with full noninductive operation has been obtained. Underlying this work are studies validating approaches to confinement extrapolation, disruption avoidance and mitigation, tritium retention, ELM avoidance, and operation above the no-wall pressure limit. In addition, the unique capabilities of the DIII-D facility have advanced studies of the sawtooth instability with unprecedented time and space resolution, threshold behavior in the electron heat transport, and rotation in plasmas in the absence of external torque.
\end{abstract}

\section{Introduction}

The DIII-D Team has made significant progress in its stated mission to establish the scientific basis for optimization of the tokamak approach to fusion energy production. The DIII-D program seeks to provide the necessary basis for the operational scenarios and to validate design models for areas of significant concern in next-generation tokamaks for the study of burning plasmas (ITER) and steady-state issues (KSTAR, EAST). In addition, the DIII-D facility has developed into a unique research tool for investigation of hightemperature plasma physics due to the flexibility of the tokamak itself, the variety of heating and current drive systems, and the continually improving ability to diagnose plasmas. Burning plasma issues, steady-state scenario development, and basic plasma physics set the outline for this overview of recent results from the DIII-D Program.

\section{Burning Plasma Scenario Development}

\subsection{Proof of Performance in Stationary Discharges}

A central element of DIII-D research in support of burning plasma experiments is a demonstration of performance in stationary discharges. The emphasis on stationary discharges arises from the question of whether high performance discharges achieved transiently can be extended reliably to long pulse. The longest plasma physics time scale in the tokamak is the time for equilibration of the current profile $\tau_{R}$. This time is defined here as the time constant for relaxation of the lowest order spatial eigenmode of the current evolution equation with the constraint of constant current [1], $\tau_{\mathrm{R}}(s) \equiv 0.171 R / \Re$, where $R$ is the plasma major radius in $m$ and $\Re$ is the plasma resistance [2] in $\mu \Omega$. In order to compare fusion performance between next-generation tokamaks, such as ITER, and DIII-D, the leading-order dimensionless parameter in the fusion gain, $G \equiv \beta_{\mathrm{N}} H_{89} / q_{95}^{2}$, is used [3]. As a benchmark, the baseline ITER design [4] requires $G=0.42$ to achieve $Q_{\text {fus }}=10$ for $\sim 2 \tau_{\mathrm{R}}$. DIII-D has demonstrated normalized fusion performance which meets or exceeds these values under stationary conditions (Fig. 1). As shown in Fig. 1, discharges in DIII-D provide a basis for confidence that the core plasma in the ITER design can meet the performance goals of the project and for optimism that such a device could significantly exceed those goals.

A DIII-D discharge with high performance under conditions close to the ITER baseline scenario is shown in Fig. 2. The normalized fusion performance $G=0.58$ at $q_{95}=3.2$ is

\footnotetext{
* See Appendix A for a listing of the DIII-D Team.
} 
sustained for $9.2 \tau_{\mathrm{R}}$. The normalized pressure $\beta_{\mathrm{N}}=2.6$ is maintained by feedback control of the neutral beam injection (NBI) power. The density is controlled by continuous gas puffing and pumping by cryopumps in the divertor. The details of how these discharges are formed are discussed extensively elsewhere $[5,6]$.

Direct measurement of the internal magnetic fields by motional Stark effect spectroscopy validates the definition of $\tau_{R}$, showing equilibrium of the plasma current to a truly stationary state in $<2 \tau_{R}$ with the exception of the cyclic sawtooth instability in the center. The discharge in this case is limited only by the requested duration. Projection of a similar discharge to ITER [5] provides confidence that ITER can meet or even exceed its performance target.

A second mode of operation in ITER, designed to give maximum fluence for nuclear testing, envisions an inductively-driven plasma at reduced plasma current. It is known as
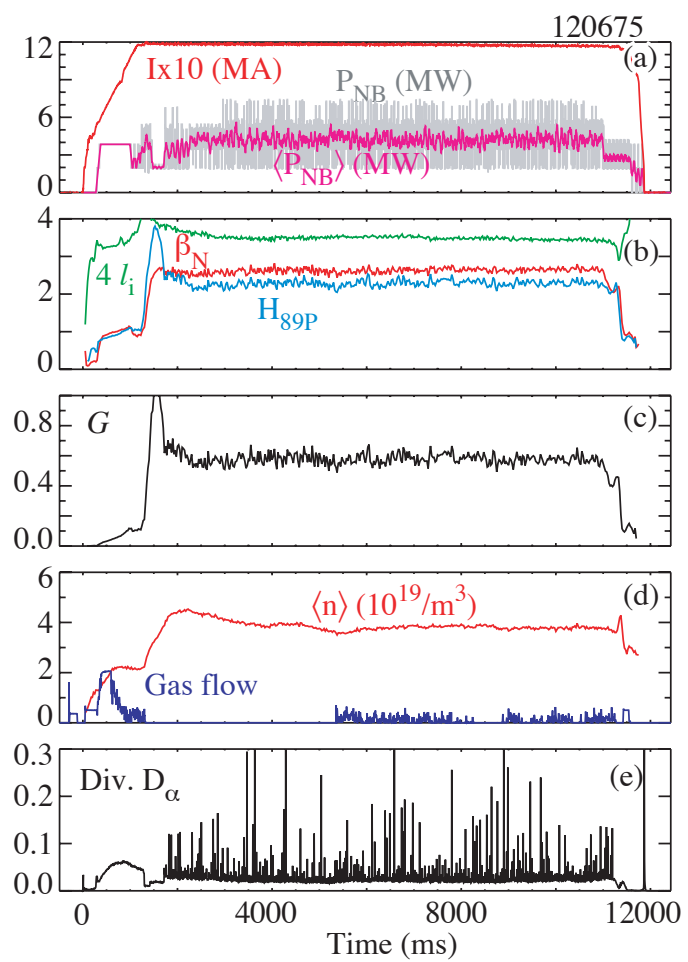

Fig. 2. Time histories of plasma parameters for an ITER baseline scenario discharge from DIII-D. (a) Plasma current (I)x10 (MA) (red), NBI power $\left(P_{\mathrm{NB}}\right)(\mathrm{MW})$ (gray), and time-averaged NBI power $\left(\left\langle P_{\mathrm{NB}}\right\rangle\right)(\mathrm{MW})$, (b) normalized pressure $\left(\beta_{\mathrm{N}}\right)$ (red),normalized energy confinement $\left(H_{89}\right)$ (blue), internal inductance $\left(l_{\mathrm{i}}\right) \times 4$ (green), (c) normalized fusion performance $(G)$, (d) lineaveraged density $(\langle n\rangle)\left(10^{19} \mathrm{~m}^{-3}\right)$ (red), $\mathrm{D}_{2}$ gas flow (a.u.) (blue), (e) upper divertor $\mathrm{D}_{\alpha}$ (a.u.).

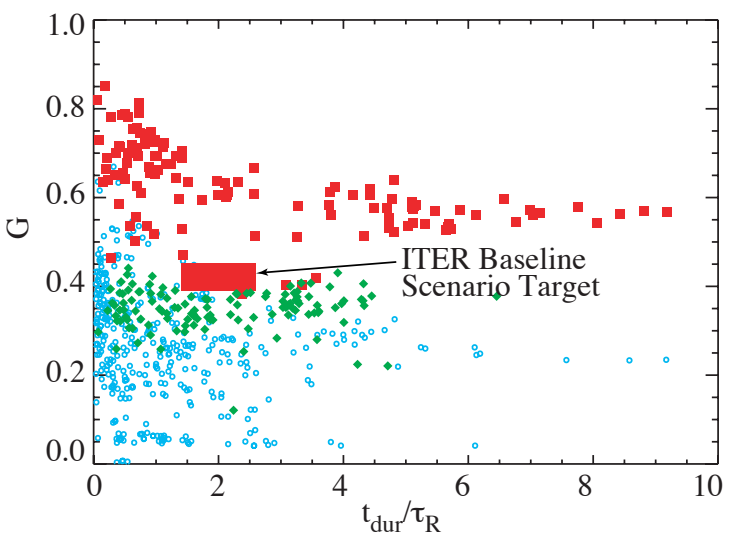

Fig. 1. Normalized fusion performance $G$ vs. duration normalized to current relaxation time. Filled squares (red) are ITER baseline scenario discharges, filled diamonds (green) are hybrid scenario discharges, open circles (light blue) are other types. 
"hybrid" operation, since the current drive systems would be employed to extend the pulse length, but without the constraints of full noninductive operation. DIII-D discharges denoted as hybrid discharges in Fig. 1 indicate the baseline performance goals of ITER could be realized for pulse lengths in excess of 1 hour at reduced plasma current (Table I), in addition to enabling a nuclear testing program. An example of this type of pulse is shown in Fig. 3. The normalized fusion performance $G=0.43$ is maintained at $q_{95}=4.1$ for $3.9 \tau_{\mathrm{R}}$. Higher $\beta_{\mathrm{N}}$ has been achieved in similar stationary discharges $[5,6]$. Remarkably, these discharges reach stationary current profiles without sawtooth activity. A modest $m=3 / n=2$ tearing mode appears to be responsible for the broadening of the current profile to keep $q=1$. Suppression of this mode by electron cyclotron current results in prompt sawtooth instability [6], which leads to reduced performance due to a lower pressure limit.

The main benefit of these discharges is a higher pressure limit. The effective pressure limit in both scenarios is set by destabilization of the $m=2 / n=1$ tearing mode. Following a request from the ITPA, the existence domain of this type of discharge has been explored in DIII-D. Pressure limits in stationary discharges have been found for $3.2<q_{95}<4.8$ and $0.3<n / n_{\mathrm{G}}<0.7$ (Fig. 4). In general, the $\beta$ limits are lower in sawtoothing discharges $\left(q_{95}<4\right)$, while confinement is best at low density. The normalized fusion performance is maximum at lowest $q_{95}$ because $\beta_{\mathrm{N}} H$ is roughly constant within the two classes of discharges (sawtoothing or sawtooth free). Between Fig. 1 and Fig. 4, it is clear that this type of discharge is robustly attainable. The physics of these high performance discharges is discussed more extensively in $[5,6]$.

\subsection{Projection of Energy Confinement to Next-Generation Tokamaks}

In the absence of a complete predictive model for the transport of particles, momentum, and energy in the tokamak, empirical scaling relations are used to project the performance of present-day experiments to burning plasmas. The projections of Table I point out the variability in this exercise, especially at high $Q_{\text {fus }}$. Dedicated experiments in DIII-D and JET have recently focused on the scaling of energy
TABLE I. Projection of hybrid discharge to ITER. The input parameters for the projection are $\mathrm{q}_{95}=4.1(\mathrm{I}=10.8 \mathrm{MA}), \beta_{\mathrm{N}}=2.6, n / n_{\mathrm{G}}=0.85, \mathrm{~B}=$ 5.3 T. Measured DIII-D temperature and density profiles are used. The three confinement scalings are the original ITER L-mode scaling (ITER89P) [7], the ITER Physics Basis H-mode scaling (IPB98y2) [8], and a fit to the ITER database with gyroBohm and electrostatic scalings imposed (DS03) [9]. The $\mathrm{H}$ factor is the DIII-D value except for DS03, where it must be reduced to achieved pressure balance (experimental value in parentheses). Flux consumption calculations indicate ITER pulse duration $>3900 \mathrm{~s}$ is possible.

\begin{tabular}{llccc}
\hline & \multicolumn{3}{c}{$\mathrm{P}_{\text {fus }}$} & $\mathrm{P}_{\text {aux }}$ \\
& $(\mathrm{MW})$ & $(\mathrm{MW})$ & $Q_{\text {fus }}$ \\
\hline ITER89P & 2.75 & 440 & 49 & 9.0 \\
IPB98y2 & 1.59 & 440 & 49 & 9.0 \\
DS03 & 1.78 & 370 & 0 & $\infty$ \\
& $(1.81)$ & & & \\
\hline
\end{tabular}

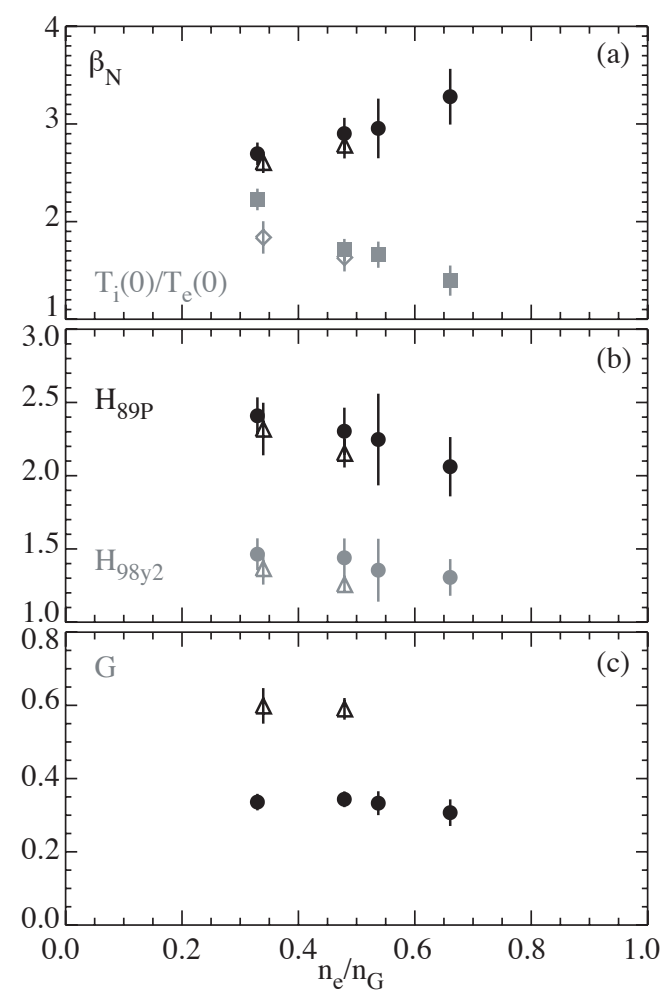

Fig. 4. Performance indices for low $q_{95}$ (3.2) (open symbols) and high $q_{95}$ (4.5) (solid symbols) for DIII-D hybrid scenario discharges vs density normalized to the empirical density limit $n_{\mathrm{G}}$ (a) $\beta_{\mathrm{N}}$ (black), $T_{\mathrm{i}}(0) / T_{\mathrm{e}}(0)$ (gray), (b) $H_{89 \mathrm{P}}$ (black), $H_{98 y 2}$ (gray), (c) $G$. All points shown are at the maximum obtained $\beta$ for stationary discharges. 
transport with $\beta$. The standard $\mathrm{H}$-mode thermal confinement scaling (known as IPB98y2) has a substantial degradation with $\beta$ [8], while direct measurements of the $\beta$ scaling in dedicated experiments showed no variation of confinement with $\beta[10,11]$. These scans have recently been reproduced and extended in both DIII-D [12] and JET [13]. As shown in Fig. 5, the observed energy confinement in $\mathrm{H}$-mode is independent of $\beta$ over the range from just above the $\mathrm{H}$-mode transition threshold to $85 \%$ of the ideal ballooning limit (nearly a factor of three in $\beta$ in the DIII-D case). The spurious $\beta$ dependence in the IPB98y 2 has a significant impact on optimizations of tokamak plasmas. The degradation of confinement with increasing $\beta$ is so large in the IPB98y2 scaling that $Q_{\text {fus }}$ actually is predicted to drop with increasing $\beta$ (Figs. 13 and 14 of [12]). This persistent discrepancy between the dimensionless parameter scaling experiments and the $\mathrm{H}$-mode database analysis has led to a significant re-examination of the analysis techniques used to derive the IPB98y2 scaling, leading to reduction of the $\beta$ scaling inferred from the database [14].

\subsection{Disruption Avoidance and Mitigation}

The total free energy stored in the poloidal magnetic field and pressure of fullperformance ITER discharges is on the order of 1 GJ. If released suddenly, damage to the first wall or vacuum vessel structure could occur. Putting aside mechanical failures for the moment, disruptions of tokamak plasmas are the result of approaching known operational boundaries such as high current, high density, or high pressure. The first two boundaries are easily avoided. The high pressure boundary manifests itself in conventional discharges as an $m=2 / n=1$ tearing mode. In the absence of rotation between the mode and the conducting wall, the mode can grow rapidly and lead to a disruption. As a first line of defense against this type of disruption, electron cyclotron current drive (ECCD) has been employed to eliminate $m=2 / n=1$ tearing modes at $\beta_{\mathrm{p}}>1$ (strongly bootstrap-driven modes). The effectiveness of the suppression is greatly enhanced by the application of active feedback to co-locate the ECCD with the mode [15]. Using active feedback, $\mathrm{m}=2 / \mathrm{n}=1$ tearing modes have been suppressed in hybrid scenario discharges at high $\beta$ $\left(\beta_{\mathrm{N}}=2.8, \beta_{\mathrm{p}}=1.1\right)$ with ECCD (Fig. 6). Experiments comparing co-ECCD, ECH, and ctr-

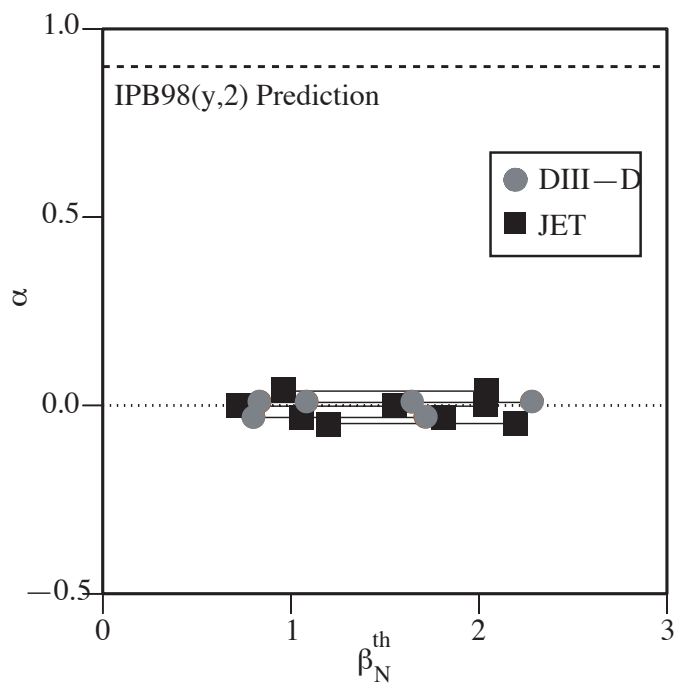

Fig. 5. Energy confinement scaling exponent $\left(\tau_{\mathrm{E}} \propto \beta^{-\alpha}\right)$ vs. normalized thermal pressure $\left(\beta_{\mathrm{N}}^{\text {th }}\right)$ for DIII-D and JET scans with fixed $\rho_{*}, \nu_{*}$, and $q$. The dimensionless parameters other than $\beta$.
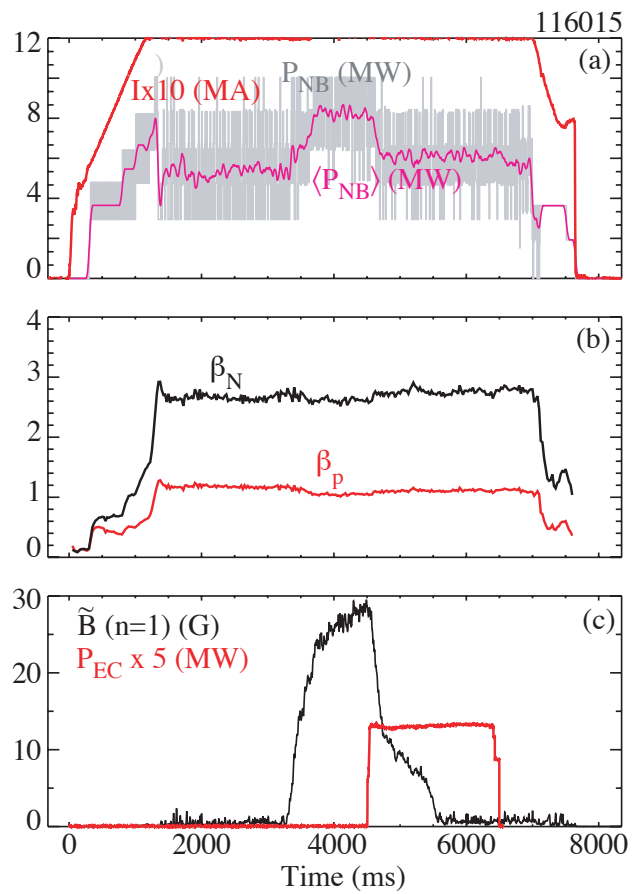

Fig. 6. Time histories of plasma parameters for a discharge where an $m=2 / n=1$ tearing mode is suppressed by the addition of ECCD at the island location. (a) Plasma current (I)x10 (MA) (red), NBI power $\left(P_{\mathrm{NB}}\right)(\mathrm{MW})$, time-averaged NBI power $\left(\left\langle P_{\mathrm{NB}}\right\rangle\right)(\mathrm{MW}),(\mathrm{b})$ normalized pressures $\beta_{\mathrm{N}}$ (black) and $\beta_{\mathrm{p}}$ (red), (c) amplitude of $\mathrm{n}=1$ magnetic perturbation at the wall $(G)$ (black), EC power $\left(P_{\mathrm{EC}}\right) \times 5(\mathrm{MW})$. points connected by lines represent scans with fixed 
ECCD show conclusively that the current drive is the dominant interaction with the mode $[16,17]$. In principle, techniques for operation above the normal instability threshold, demonstrated for the $m=3 / n=2$ tearing mode [18], could be applied to the $m=2 / n=1$ for performance enhancement. High reliability would be necessary in the context of a burning-plasma tokamak.

The next line of defense against all types of disruptions, including events separate from plasma physics limits, would be a system to dissipate the free energy in a controlled manner with more uniform power flux to the walls. One approach, injection of high-pressure gas, has been successfully demonstrated in DIII-D [19]. Measurements of the divertor energy flux during a disruption shows both significant reduction and more uniform deposition of the energy with gas jet mitigation, compared to an unmitigated disruption. Data from DIII-D shows a high fraction of the thermal energy is converted to radiation with no clear dependence on gas pressure or working gas (neon or argon). This is consistent with the hypothesis that significant mixing occurs following the jet/plasma interaction, perhaps due to MHD instabilities. Images of the gas jet also indicate that it does not penetrate to the center of the plasma in well-mitigated disruptions, prior to this mixing phase. This implies that a gas jet in ITER may not need to penetrate to the core to successfully mitigate a disruption. Measurements of the X-ray brightness in the current quench phase of DIII-D disruptions, a qualitative measure of runaway population, indicate a strong reduction in the x-ray brightness with increased particle injection (Fig. 7). The gas jet injects about two orders of magnitude more particles into the vessel than previous impurity pellet experiments, resulting in a two order of magnitude reduction in x-ray brightness (and presumably the runaway population). Avoidance of runaways is an essential element of a practical mitigation scheme for high current discharges such as ITER. Finally, a rapid thermal quench in ITER could result in melting damage to the first wall, if beryllium is used. The thermal quench time in DIII-D appears to be a weakly increasing function of the number of injected particles, with systematic differences between neon and argon injection. A validated model of the jet/plasma interaction is needed to effectively extrapolate these observations to ITER. In summary, the DIII-D data indicate that noble gas injection is a viable candidate for disruption mitigation in nextgeneration tokamaks such as ITER.

\subsection{Interaction of Plasma With the First Wall}

Routine operation of H-mode discharges with edge localized mode (ELMs) may have a significant impact on the divertor lifetime in ITER. In an ELM event, the impulsive thermal loads to the divertor can be up to $20 \%$ of the H-mode pedestal energy [20]. Because this energy is deposited in a very short time, the divertor plate surface temperature could rise to the melting or sublimation point within a single ELM event. Without a fully predictive model for the ELM energy loss and its transport on open field lines to the divertor or the main chamber, it seems prudent to investigate methods for suppressing ELMs. In DIII-D, a set of

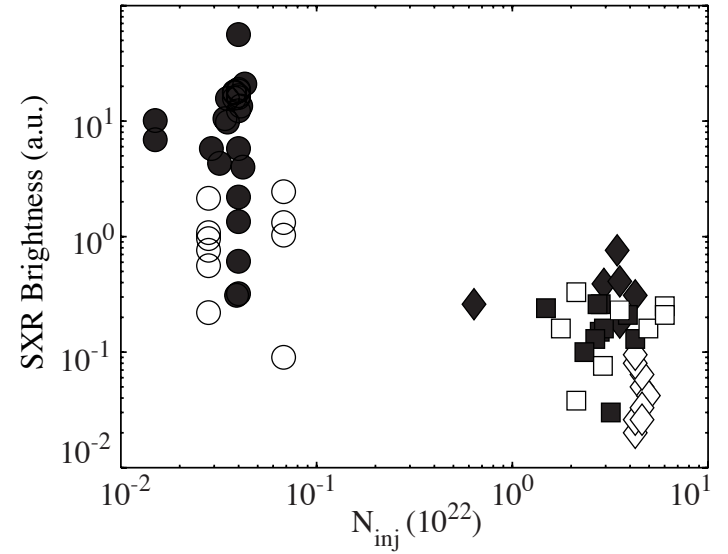

Fig. 7. X-ray brightness vs. number of injected particles for argon (solid symbols) or neon (open symbols). The circles are pellet injection, the squares are gas jet injection with a large-angle jet, and the diamonds are gas jet injection with a narrow-angle jet.

been installed inside the vacuum vessel above and below the midplane. When energized with $n=3$ symmetry during H-mode discharges with Type I ELMs, the large ELM behavior is replaced by smaller, less distinct instabilities [21]. without loss of energy confinement (Fig. 8). The impulsive thermal load can be reduced by at least a factor of 3 . The application 
of the non-axisymmetric field is correlated with a shift in physical space of the measured electron and ion pressure profiles with little change in gradient or pedestal height. It is difficult to determine whether the profiles shift with respect to the meanfield separatrix, although the fact that the electron and ion profiles are measured at different toroidal and poloidal locations makes this plausible. The change in edge stability is correlated with this shift, but again it is difficult to separate cause and effect. Field line tracing indicates the formation of two stochastic field regions, one contained in the plasma and one connecting the outer $3 \%$ of the flux enclosed within the mean-field separatrix to the wall. From variations of the toroidal phase of the applied perturbation, it appears that there is significant interaction with the intrinsic nonaxisymmetric fields in DIII-D. From energy accounting, the losses across the separatrix still flow to the dominant divertor. These experiments show that it is possible to avoid the negative effects of ELMs while maintaining a significant edge pressure pedestal.

The choice of first-wall materials may also have an impact on routine operations. Graphite is the material with the largest experience basis; however, retention of tritium due to co-deposition with carbon could lead to an excessive inventory of tritium in a next-generation device. Experiments in DIII-D are focused on gaining an understanding of the carbon migration, leading to methods of reducing the bound tritium inventory either by improved design or more effective removal of the deposited tritium. Using ${ }^{13} \mathrm{C}$ as a tracer to follow the migration of carbon [22], doped methane gas was introduced with toroidal symmetry into the scrape-off layer (SOL) opposite the X-point of a L-mode diverted plasma [23]. Visible light images of the gas plume indicated that the ionized carbon in the SOL had a strong flow in the direction of the inner divertor leg. Immediately following the 22 similar discharges with ${ }^{13} \mathrm{C}$ injection, a poloidal band of graphite tiles was removed for analysis. Deposited ${ }^{13} \mathrm{C}$ was found only in the inner divertor region at the SOL contacts. Two tiles from the inner and outer divertor strikepoint region displaced $180 \mathrm{deg}$ toroidally showed an exact match of the detected ${ }^{13} \mathrm{C}$, indicating the deposition was also axisymmetric. Modeling of the observed plumes, the ${ }^{13} \mathrm{C}$ deposition, and the change in the core plasma carbon inventory indicated that all are sensitive to the magnitude of the assumed SOL flow. The best match of the modeling with the experiment is obtained with a parallel flow with Mach number $\sim 0.4$ in the direction of the inner divertor. Modeling also indicates a flow in the private flux region from the outer to inner leg. Since the carbon migration (and therefore the tritium inventory) is strongly focused to a single poloidal location, techniques to release tritium bound in this single area may be more feasible compared with the situation where tritium is more widely distributed within the tokamak. 


\section{Steady-State Scenario Development}

\subsection{Proof of Performance of Full Noninductive Discharges}

Looking beyond the next generation of tokamaks to fusion energy production, a key issue is the capability of the tokamak concept to operate with high $Q_{\text {fus }}$ in steady state. Research in DIII-D has addressed this issue over the past decade, and significant progress has been made in the past two years toward an existence proof of a tokamak discharge sustained noninductively that projects to high $Q_{\text {fus }}$ in a tokamak the size of ITER. Previously,discharges were reported that had no net inductive current [24]; however, the noninductive currents were not fully aligned with the existing current profile, as indicated by the structure in the measured electric field profile. Recently, discharges with much better alignment have been obtained with nearly flat electric field profiles and no change in the boundary flux [25]. An example shown in Figs. 9 and 10 has $\beta_{\mathrm{N}} \leq 3.5, \mathrm{H}_{89}=2.3$, and $q_{95}=5.4$. The high performance phase lasts $\sim 0.8 \mathrm{~s}$ and is terminated by an $n=1$ ideal mode that transforms to an $n=1$ tearing mode. Projection of this discharge to ITER, using the same method as the baseline and hybrid scenario discharges, results in discharges limited by the confinement to $\beta_{\mathrm{N}}=2.8$ with the specified $73 \mathrm{MW}$ of auxiliary power (33 MW
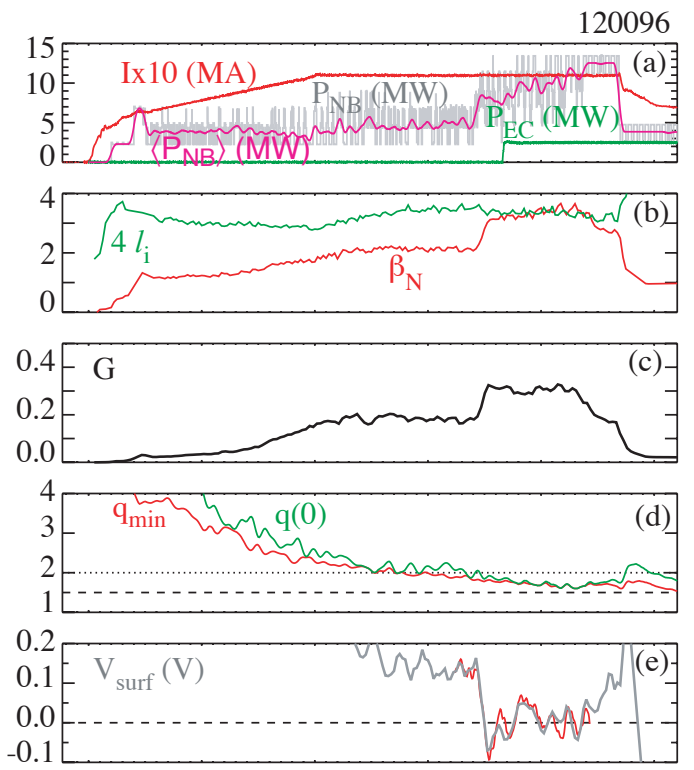

0.1

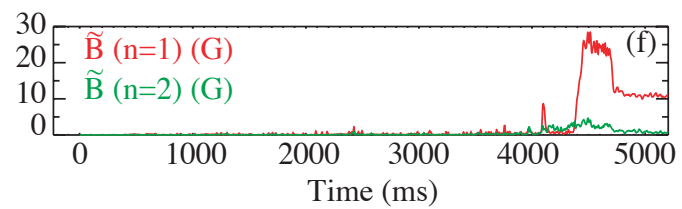

Fig. 9. Time histories of plasma parameters for a full noninductive discharge in DIII-D which projects to $Q_{\text {fus }} \sim 5$ in ITER. (a) Plasma current (I)x10 (MA) (red), NBI power $\left(P_{\mathrm{NB}}\right)(\mathrm{MW})$ (gray), time-averaged NBI power $\left(\left\langle P_{\mathrm{NB}}\right\rangle\right)$ (MW) (magenta), EC power (MW) (green), (b) normalized pressure $\left(\beta_{\mathrm{N}}\right)$ (red), internal inductance $\left(l_{\mathrm{i}}\right) \mathrm{x} 4$ (green), (c) normalized fusion performance $(G)$, (d) minimum safety factor $\left(q_{\text {min }}\right)$ (red), central safety factor $[q(0)]$ (green), (e) surface voltage (gray), surface voltage from high time resolution equilibrium reconstructions (red) (V), (f) magnetic fluctuations at the wall $(G)$ for $n=1$ (red) and $n=2$ (green).

N-NBI, $20 \mathrm{MW}$ EC, $20 \mathrm{MW}$ IC). The 0-D current balance is satisfied at $9.3 \mathrm{MA}$ with $Q_{\text {fus }}=$ 4.7, slightly below the target of $Q_{\text {fus }}=5$ (Table II). To increase $Q_{\text {fus }}$, the plasma current must be increased. Assessing the stability of flat $q$ profiles as a function of $q_{95}$ and $q_{\min }$ remains an important goal [26].
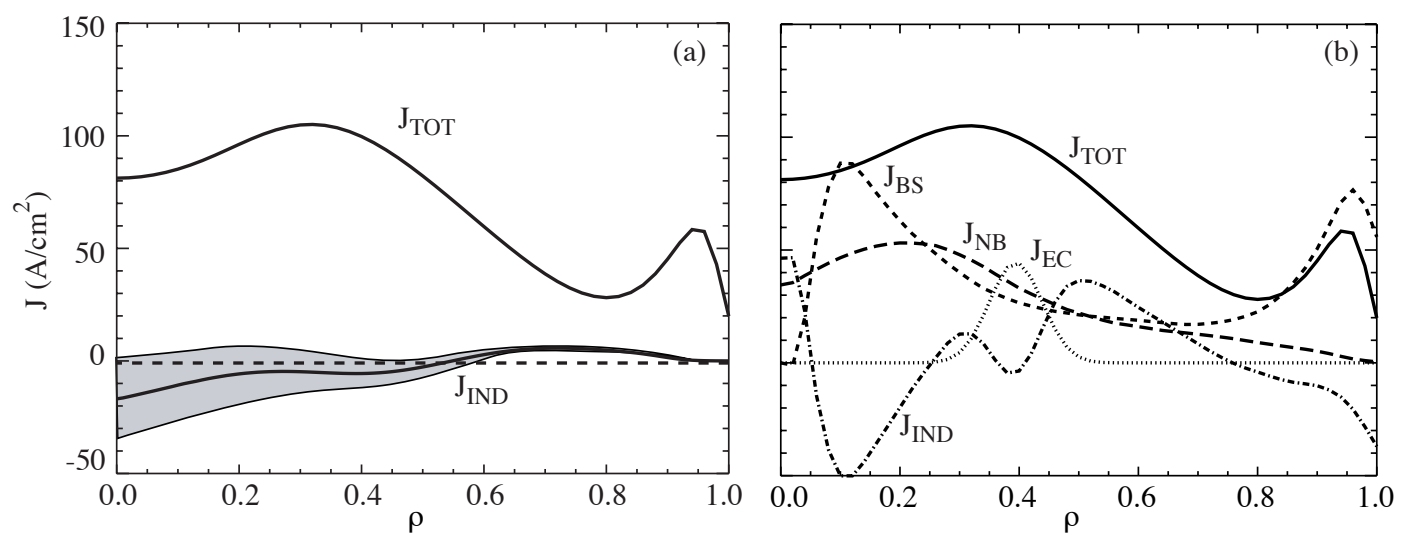

Fig. 10. (a) Total current density (solid) and inductive current density (shaded) inferred from equilibrium reconstruction vs. normalized radius. (b) Components of the current density vs. normalized radius from modeling. Total (solid), NB (long dashed), bootstrap (short dashed), EC (dotted), balance (attributed to inductive) (dot-dashed). 
The fact that the discharge in Fig. 9 still encounters a tearing instability indicates the internal current profile is still slowly evolving despite no net inductive flux.The good alignment of the noninductive current sources with the total current density is illustrated in Fig. 10(a) by the flat distribution of the inductive current inferred from the time dependence of the poloidal flux from equilibrium reconstructions. Taking the measured profiles and reconstructed equilibria, the components of the noninductive current can be calculated from various models [Fig. 10(b)]. The bootstrap current fraction is estimated to be 0.58 using the NCLASS model, with other models giving similar fractions. The models are somewhat sensitive to the input profiles near both the center and edge. The neutral beam current fraction is estimated at 0.31 , while the electron cyclotron current fraction is 0.07 , giving a total noninductive fraction from modeling of 0.96. The remaining current in the model calculation is attributed to inductive current. Comparison of the inductive currents in Fig. 10 indicates more structure in the inductive current inferred from the modeling than in the inductive current inferred from equilibrium reconstructions. Since the poloidal flux is fairly accurately determined and its evolution is diffusive with a long time constant ( $2 \mathrm{~s}$ in this case), the inductive current profile in Fig. 10(a) would seem to be a better estimate of the remaining inductive current. All of these methods of determining the various components of the current density indicate that a fully noninductive discharge with high performance has been obtained.

\subsection{Stabilization of Resistive Wall Modes}

Self-consistent steady-state scenarios generally require operation above the $n=1$ ideal MHD pressure limit in the absence of a conducting wall. With a resistive wall, a new mode appears, the resistive wall mode (RWM), which is unstable, but with a growth time on the order of the skin time of the resistive wall [27]. As previously shown [28], plasma rotation is sufficient to stabilize this mode up to the perfectly conducting wall limit. In the absence of sufficient rotation, this mode is amenable to active feedback control due to the slow growth rate [29]. There is also a self-consistent plasma response to any non-axisymmetric external magnetic perturbations that acts to drag down the rotation, thereby increasing the growth rate of the mode [30]. Correction of these perturbations using feedback has been demonstrated to facilitate the rotational stabilization of the RWM [31]. Because the main heating system in DIII-D (NBI) also supplies torque, plasma rotation is sufficient to stabilize many DIII-D discharges.

Recently, discharges have been obtained in DIII-D where the $n=1$ RWM is not stabilized by rotation. However, the addition of active feedback with the new internal coils [32] allows the pressure to continue to rise without destabilizing the RWM (Fig. 11). In both cases, the rotation is above the empirical critical rotation for stabilization, found in previous experiments. The difference in the present discharges appears to be lower $q_{95}$, which reduces the number of low-order rational surfaces in the plasma core. Theoretical calculations also predict a higher rotation is necessary for stabilization when fewer rational surfaces are present [33]. Development of these target discharges will allow optimization of the active feedback of the RWM. 


\section{Basic Physics Studies}

Continuing improvement in diagnostics, coupled with the flexibility of the DIII-D tokamak and heating systems, supports an extensive program of basic tokamak physics experiments on DIII-D. Here, the focus will be on plasma instabilities and transport.

\subsection{Sawtooth Physics}

Sawtooth instabilities are ubiquitous in tokamaks. However, a detailed theoretical description of the associated phenomena has proven elusive. A key issue is the role of the Mercier criterion relative to $q=1$. It is expected that regions of the plasma that violate the Mercier criterion will have no pressure gradient, while discharges with a $q=1$ surface will be unstable to an internal kink. In DIII-D, it is possible to move the Mercier criterion above or below $q=1$ by changing the discharge poloidal crosssection. Oval shapes place the Mercier criterion at $q>1$ for standard profiles, while indented shapes place the Mercier criterion at $q<1$ [34].

The sawtooth instability has been observed with sub-millisecond resolution in the electron temperature, the ion kinetic parameters, and the magnetic pitch angle for both cases (Fig. 12). Clear differences in the instability are observed in the two cases. For the oval, the current profile does not strongly evolve in between crashes, consistent with the poloidal magnetic field inside the inversion radius shown in Fig. 12. The reconstructed equilibria have flat $q$ profiles with $q \sim 1$. In the indented case, $q(0)$ drops well below $q=1$ during the sawtooth cycle, again consistent with the large excursions in the poloidal magnetic field shown in Fig. 12. In order to test the ability of the plasma to support a pressure gradient, single pulses of ECH were placed inside the sawtooth inversion radius. In the indented case, the electron temperature gradients strongly increased, while in the oval case, very little temperature increase was seen despite a 25 -fold increase in local power density (Fig. 13). This is consistent with the basic idea of the Mercier limit. However, there is still an ion temperature gradient in the oval case, which raises questions about the applicability of ideal MHD in this case. Detailed comparison to theory is still in progress.

\subsection{Search for Threshold Behavior in Electron Energy Transport}

The existence of threshold behavior or critical gradients in the stability of the microturbulence responsible for anomalous heat transport in tokamaks is a significant issue in evaluating the validity of various theories. Using experimental approaches developed on 
ASDEX-Upgrade (AUG) and FT-U, modulated ECH experiments on DIII-D have probed L-mode discharges for thresholds in the electron heat transport. An enhancement in the heat pulse evolution above that expected for pure diffusion at the power balance level is observed, but no direct evidence of a threshold is seen. In the AUG type of experiment, the power at two spatially separated ECH locations is varied, keeping the total power fixed. Modulation of a fraction of the power is used to estimate the transport from the heat pulse evolution. Reduction of the inverse gradient scale length $\left(-\nabla T_{\mathrm{e}} / T_{\mathrm{e}}\right)$ down to 3.8 in DIII-D and 2.6 in AUG does not change the heat pulse behavior (Fig. 14). While the data can be described by a model with a critical gradient, the data does not compel the use of such a model [35]. A more direct probe of nonlinearity of the electron transport is the "swing" method developed on FT-U. The idea is to vary the temperature gradients strongly at a fixed radius and temperature by oscillating two spatially separated $\mathrm{ECH}$ sources out of phase. If there is a strong nonlinearity in the heat transport, the resultant heat pulse behavior at the point between the two sources should be different from the sum of the individual pulses. In DIII-D, nonlinear behavior is not observed down to $-\nabla T_{\mathrm{e}} / T_{\mathrm{e}}=2.5$. If there is a threshold condition for the electron transport, it lies at gradients less than those near the center of low-power L-mode plasmas. Therefore, the threshold is always exceeded for the plasmas probed in DIII-D. Furthermore, the gradients can be increased by more than a factor of 2 , which excludes the possibility of very stiff transport.

\subsection{Studies of Plasma Rotation}

Rotation is a significant unknown for both confinement and stability predictions for next-generation tokamaks; yet, in comparison to energy transport, little experimental or theoretical work has been done.
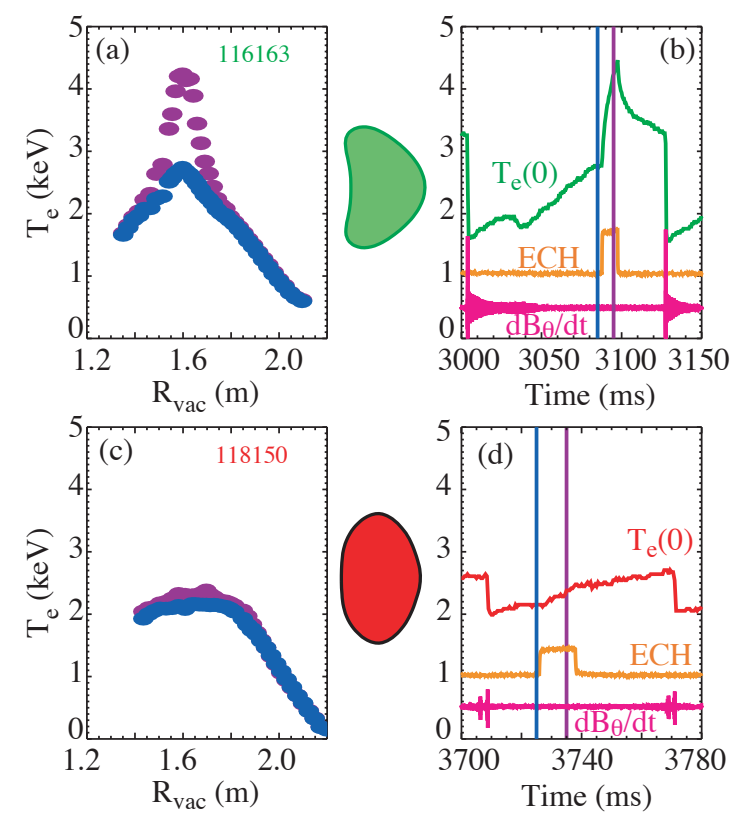

Fig. 13. Radial profiles of the electron temperature (left boxes) and time histories (right boxes) of the central electron temperature $T_{\mathrm{e}}(\mathrm{keV})$ showing the effects of applying ECH within the inversion radius during the rise phase of the sawtooth cycle for indented (upper) and oval (lower) cross-section plasmas. The right boxes also show the EC power timing (yellow) and magnetic fluctuations measured at the wall (magenta). The profiles in the left boxes are from the times designated by the color-matched vertical lines in the right boxes.

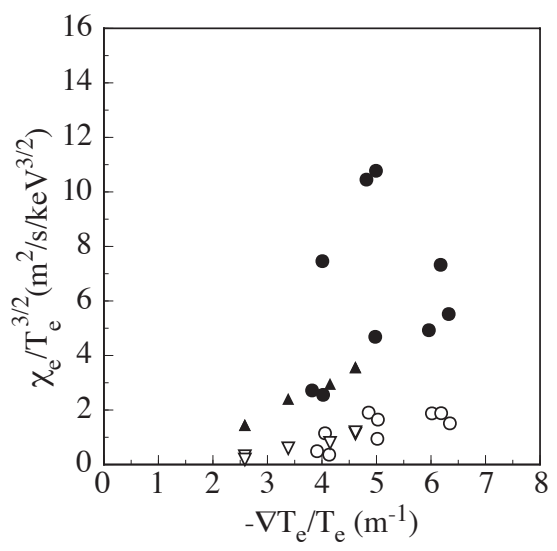

Fig. 14. Normalized electron diffusivity vs. the temperature gradient normalized to temperature for DIII-D (circles) and AUG (triangles) discharges. The open symbols are diffusivities derived from power balance analysis and the filled symbols are diffusivities derived from modulated ECH analysis.

New measurements in DIII-D of rotation in

Ohmic H-mode [36] show flat toroidal rotation profiles with direction consistent with an electric field generated by the loss of thermal ions at the edge with parallel velocity opposite the plasma current direction. In the absence of momentum sources, a flat profile is expected. The level of rotation is qualitatively consistent with the flows inferred from the ${ }^{13} \mathrm{C}$ experiments discussed above. When ECH is added on-axis or off-axis, a hollow rotation 
profile is observed. Since no torque is applied, the mechanism for this modification of the profile is unknown. Possibilities include non-ambipolar particle transport or a change in the ion pressure anisotropy. The direction of the change in rotation with $\mathrm{ECH}$ is opposite that in central ICRH discharges in Alcator C-Mod [37].

\section{Conclusions}

The DIII-D facility has yielded results that substantially bolster the design basis for nextgeneration tokamaks addressing burning plasma physics issues and steady-state scenarios. Stationary discharges similar to the ITER baseline performance scenario have performance nearly $50 \%$ higher than that required to meet ITER's performance target $\left(Q_{\text {fus }}=10\right)$. Discharges focused on maximizing fusion energy in long-pulse inductive scenarios ("hybrid" scenarios) have achieved performance that is equivalent to the target $Q_{\text {fus }}=10$ conditions, which, if reproduced in ITER, could be sustained for well over one hour. The basis for these extrapolations has been more firmly established by joint experiments targeted at discrepancies between database analysis and dedicated experiments. In concert with JET, the scaling of energy confinement has been definitively determined to be independent of $\beta$, leading to improvements in the database analysis techniques. Techniques to avoid disruption by control of resistive instabilities using ECCD and to mitigate the effects of disruptions by massive noble gas injection have been successfully demonstrated. Two examples of ELM avoidance (stochastic fields near the edge) have been demonstrated. Experiments on carbon migration indicate that the majority of tritium in a graphite-wall tokamak will be near the inner divertor leg, which may ease the difficulty of removing this tritium.

A steady-state scenario that projects to significant fusion gain in ITER $\left(Q_{\text {fus }} \sim 5\right)$ has been demonstrated in DIII-D. Full noninductive operation is obtained with $\sim 60 \%$ bootstrap current, plus central NBCD and off-axis ECCD. True steady-state was not quite obtained, limited by the appearance of an instability after about $0.8 \mathrm{~s}$. This indicates a higher degree of control is required. However, the demonstration of full noninductive operation on the timescale of pressure equilibration $\left(\sim 5 \tau_{\mathrm{E}}\right)$ that projects to high gain is a significant achievement. The noninductive current profile is closely matched to the total current profile, indicating a small adjustment should be sufficient to reach stationary conditions. Full noninductive discharges at higher $q_{95}$ without the constraint of constant current indicate that these conditions may not be a stationary solution to the coupled fluid equations. Experiments with feedback control of the RWM that appears above the $n=1$ no-wall limit have shown the effectiveness of rotational and active feedback.

Upgrades to the DIII-D diagnostic suite have opened new windows on basic tokamak physics. Combination of high time-resolution measurements, the unique shaping capabilities of DIII-D, and the possibility of ECH have led to a demonstration of the importance of the Mercier criterion to the character of the sawtooth instability. These same tools have been applied to the search for threshold behavior in the electron heat transport and to studies of plasma rotation in the absence of external torque. In the case of the threshold, no direct evidence of threshold behavior is seen, even in low-power L-mode plasmas. Therefore, if such a threshold exists, it is always exceeded in the plasmas examined. For rotation, a clear change in rotation direction is observed in $\mathrm{H}$ mode with the application of $\mathrm{ECH}$.

\section{Acknowledgment}

This work was supported by the U.S. Department of Energy under Cooperative Agreement DE-FC02-04ER54698 and UC LLNL contract W-7405-Eng-48. 


\section{References}

[1] MIKKELSEN, D.R., Phys. Fluids B 1 (1989) 333.

[2] SAUTER, O., et al., Phys. Plasmas 6 (1999) 2834.

[3] LUCE, T.C., "Advanced tokamak research in DIII-D," submitted to Fusion Sci. Tech. (2004).

[4] AYMAR, R., et al., Plasma Phys. Control. Fusion 44 (2002) 519.

[5] LUCE, T.C., et al., Phys. Plasmas 11 (2004) 2627.

[6] WADE, M.R., et al., this conference, submitted to Nucl. Fusion.

[7] YUSHMANOV, P.N., et al., Nucl. Fusion 30 (1990) 1999.

[8] ITER Physics Basis, Nucl. Fusion 39 (1999) 2175.

[9] PETTY, C.C., et al., Fusion Sci. Tech. 43 (2003) 1.

[10] PETTY, C.C., et al., Nucl. Fusion 38 (1998) 1183.

[11] CORDEY, J.G., et al., in Fusion Energy 1996 (IAEA, Vienna, 1997) Vol. 1, p. 603.

[12] PETTY, C.C., et al., Phys. Plasmas 11 (2004) 2514.

[13] McDONALD, D.C., et al., Plasma Phys. Control. Fusion 46 (2004) A215.

[14] CORDEY, J.G., this conference, submitted to Nucl. Fusion (2004).

[15] PETTY, C.C., et al., Nucl. Fusion 44 (2004) 243.

[16] LUCE, T.C., et al., to be published in Proc. of 13th Joint Workshop on Electron Cyclotron Emission and Electron Cyclotron Heating, Nizhny Novgorod, Russia, 2004.

[17] PETTY, C.C., et al., in Fusion Energy 2004.

[18] LA HAYE, R.J., et al., Proc. 31st EPS Conf. on Control. Fusion and Plasma Phys., London, United Kingdom, 2004 (ECA, Geneva, 2004).

[19] HOLLMANN, E.M., et al., this conference, submitted to Nucl. Fusion.

[20] LEONARD, A.W., et al., Phys. Plasmas 10 (2003) 1765.

[21] EVANS, T.E., et al., Phys. Rev. Lett. 92 (2004) 235003.

[22] LIKONEN, J., et al., Fusion Eng. and Design 66 (2003) 58.

[23] ALLEN, S.L., et al., to appear in J. Nucl. Mater. (2004).

[24] GREENFIELD, C.M., et al., Phys. Plasmas 11 (2004) 2616.

[25] MURAKAMI, M., et al., this conference, submitted to Nucl. Fusion (2004).

[26] FERRON, J.R., et al., this conference.

[27] BONDESON, A., and WARD, D.J., Phys. Rev. Lett. 72 (1994) 2709.

[28] GAROFALO, A.M., et al., Phys. Rev. Lett. 89 (2002) 235001.

[29] OKABAYASHI, M., et al., Phys. Plasmas 8 (2001) 2071.

[30] BOOZER, A.H., Phys. Rev. Lett. 86 (2001) 5059.

[31] GAROFALO, A.M., et al., Nucl. Fusion 42 (2002) 1335.

[32] STRAIT, E.J., et al., Phys. Plasmas 11 (2004) 2505.

[33] WARD, D.J., and BONDESON, A., Phys. Plasmas 2 (1995) 1570.

[34] LAZARUS, E.A., et al., this conference.

[35] DEBOO, J.C., et al., this conference, submitted to Nucl. Fusion (2004).

[36] deGRASSIE, J.S., et al., this conference.

[37] RICE, J.E., et al., Nucl. Fusion 44 (2004) 379. 


\section{APPENDIX: THE DIII-D TEAM}

G. Abla, ${ }^{1}$ S.L. Allen, ${ }^{2}$ P.M. Anderson, ${ }^{1}$ R. Andre,${ }^{3}$ G. Antar ${ }^{4}$ N. Antoniuk,${ }^{4}$ M.E. Austin, ${ }^{5}$ F.W.Baity, ${ }^{6}$ J.P. Bakalarski, ${ }^{1}$ D.R. Baker, ${ }^{1}$ D.E. Baldwin, ${ }^{1}$ D.E.G. Barber, ${ }^{6}$ R. Bastasz, ${ }^{7}$ C.B. Baxi, ${ }^{1}$ L.R. Baylor, ${ }^{6}$ M. Becoulet ${ }^{8}$ J.M. Bialek, ${ }^{9}$ J.A. Boedo, ${ }^{4}$ R.L. Boivin, ${ }^{1}$ A. Bondeson, ${ }^{10}$ R.V. Bravenec, ${ }^{5}$ B.D. Bray, ${ }^{1}$ D.P. Brennan, ${ }^{11}$ S. Brezinski, ${ }^{12}$ N.H. Brooks, ${ }^{1}$ R.V. Budny, ${ }^{3}$ K.H. Burrell, ${ }^{1}$ J.R. Burruss, ${ }^{1}$ R.J. Buttery, ${ }^{13}$ O. Buzhinskij, ${ }^{14}$ J.D. Callen, ${ }^{15}$ R.W. Callis, ${ }^{1}$ G.L. Campbell, ${ }^{1}$ J.M. Candy, ${ }^{1}$ T.N. Carlstrom, ${ }^{1}$ W.P. Cary, ${ }^{1}$ T.A. Casper, ${ }^{2}$ E. Cecil, ${ }^{16}$ C.D. Challis, ${ }^{13}$ V.S. Chan, ${ }^{1}$ M.S. Chance, ${ }^{3}$ E. Chin, ${ }^{1}$ H.K. Chiu, ${ }^{1}$ M. Choi, ${ }^{1}$ M.S. Chu, ${ }^{1}$ S. Cirant, ${ }^{17}$ R.J. Colchin, ${ }^{6}$ S.K. Combs, ${ }^{6}$ J. Culver, ${ }^{18}$ J.W. Davis, ${ }^{18}$ W. Davis, ${ }^{3}$ J.S. deGrassie, ${ }^{1}$ J.C. DeBoo, ${ }^{1}$ R.D. Deranian, ${ }^{1}$ J.L. Doane, ${ }^{1}$ R.P. Doerner, ${ }^{4}$ V. Dokouka,${ }^{14}$ J. Dorris, ${ }^{11}$ E.J. Doyle, ${ }^{19}$ D.H. Edgell, ${ }^{20}$ R.A. Ellis, III, ${ }^{3}$ R.F. Ellis, ${ }^{21}$ C. Estrada-Mila, ${ }^{4}$ T.E. Evans, ${ }^{1}$ E. Feibush, ${ }^{3}$ M.E. Fenstermacher, ${ }^{2}$ J.R. Ferron, ${ }^{1}$ K.H. Finken, ${ }^{12}$ D.K. Finkenthal, ${ }^{22}$ R.K. Fisher, ${ }^{1}$ S.M. Flanagan, ${ }^{1}$ R.J. Fonck, ${ }^{15}$ C-M. Fransson, ${ }^{23}$ E. Fredd, ${ }^{3}$ S.A. Galkin, ${ }^{24}$ A.M. Garofalo, ${ }^{9}$ K.W. Gentle,${ }^{5}$ R. Gianella,${ }^{8}$ M. Gilmore,${ }^{25}$ P. Gohil, ${ }^{1}$ I.A. Gorelov, ${ }^{1}$ R.H. Goulding, ${ }^{6}$ D.S. Gray, ${ }^{4}$ M.T. Green, ${ }^{1}$ K.L. Greene, ${ }^{1}$ C.M. Greenfield, ${ }^{1}$ N.L. Greenough, ${ }^{3}$ R.J. Groebner, ${ }^{1}$ M. Groth, ${ }^{2}$ H.J. Grunloh, ${ }^{1}$ M. Graznevitch, ${ }^{13}$ S. Günter, ${ }^{26}$ D. Gupta, ${ }^{15}$ M.J. Hansink, ${ }^{1}$ R.J. Harrington, ${ }^{27}$ J.H. Harris, ${ }^{28}$ R.W. Harvey, ${ }^{29}$ R. Hatcher, ${ }^{3}$ N.C. Hawkes, ${ }^{13}$ C.C. Hegna, ${ }^{15}$ W.W. Heidbrink, ${ }^{30}$ T.C. Hender, ${ }^{13}$ F.L. Hinton, ${ }^{1}$ J. Hobirk, ${ }^{26}$ J.T. Hogan, ${ }^{6}$ C. Holcomb, ${ }^{2}$ C. Holland, ${ }^{4}$ E.M. Hollman, ${ }^{4}$ K.L. Holtrop, ${ }^{1}$ R.-M. Hong, ${ }^{1}$ J.C. Hosea, ${ }^{3}$ N. Howard, ${ }^{31}$ D.F. Howell, ${ }^{13}$ W.A. Houlberg, ${ }^{6}$ C.-L. Hsieh, ${ }^{1}$ D.A. Humphreys, ${ }^{1}$ A.W. Hyatt, ${ }^{1}$ F. Imbeaux, ${ }^{8}$ Y. In, ${ }^{32}$ K. Indireshkumar, ${ }^{3}$ A. Isayama, ${ }^{33}$ R.C. Isler, ${ }^{6}$ G.L. Jackson, ${ }^{1}$ A.M. Jacques, ${ }^{1}$ S.C. Jardin, ${ }^{3}$ R.J. Jayakumar, ${ }^{2}$ T.H. Jensen, ${ }^{1}$ T.C. Jernigan, ${ }^{6}$ E.H. Joffrin, ${ }^{8}$ R.D. Johnson, ${ }^{1}$ R. Jong, ${ }^{2}$ K. Kajiwara, ${ }^{34}$ D.H. Kaplan, ${ }^{1}$ O. Katsuro-Hopkins, ${ }^{9}$ K.M. Keith, ${ }^{1}$ A.G. Kellman, ${ }^{1}$ D.H. Kellman, ${ }^{1}$ M.A.H. Kempenaars, ${ }^{35}$ C. Kessel, ${ }^{3}$ R. Khayrutdinov, ${ }^{14}$ C. Kim, ${ }^{15}$ J.S. Kim, ${ }^{32}$ J.E. Kinsey, ${ }^{36}$ G.J. Kramer, ${ }^{3}$ S.I. Krasheninnikov, ${ }^{4}$ S.E. Kruger, ${ }^{37}$ R.J. La Haye, ${ }^{1}$ L.L. Lao, ${ }^{1}$ C.J. Lasnier, ${ }^{2}$ J.W. Latchem, ${ }^{1}$ E.A. Lazarus, ${ }^{6}$ J.-N. Leboeuf, ${ }^{19}$ R.L. Lee, ${ }^{1}$ H. Lee, ${ }^{38}$ A.W. Leonard, ${ }^{1}$ J.A. Leuer, ${ }^{1}$ Y.R. Lin-Liu, ${ }^{39}$ S. Lisgo, ${ }^{18}$ C. Liu, ${ }^{1}$ Y.Q. Liu, ${ }^{10}$ A. Loarte, ${ }^{26}$ L.L. Lodestro, ${ }^{2}$ J. Lohr, ${ }^{1}$ T.C. Luce, ${ }^{1}$ S.C. Luckhardt,${ }^{4}$ C. Ludescher-Furth, ${ }^{3}$ Y. Luo, ${ }^{30}$ J.L. Luxon, ${ }^{1}$ M.A. Mahdavi, ${ }^{1}$ J. Mailloux, ${ }^{13}$ R. Maingi, ${ }^{6}$ C.C. Makariou, ${ }^{1}$ M.A. Makowski, ${ }^{2}$ J. Mandrekas, ${ }^{40}$ J. Manickam, ${ }^{3}$ A. Manini, ${ }^{26}$ M.E. Maraschek, ${ }^{26}$ P.S. Mauzey, ${ }^{1}$ D. Mazon, ${ }^{8}$ D.C. McCune, ${ }^{3}$ B.B. McHarg, ${ }^{1}$ G.R. McKee, ${ }^{15}$ A.G. McLean, ${ }^{18}$ J.E. Menard, ${ }^{3}$ W.H. Meyer, ${ }^{2}$ D. Mikkelsen, ${ }^{3}$ D.H. Minor, ${ }^{1}$ P.K. Mioduszewski, ${ }^{6}$ C.P. Moeller, ${ }^{1}$ J.M. Moller, ${ }^{2}$ R.A. Moyer, ${ }^{4}$ A. Mui, ${ }^{22}$ M. Murakami, ${ }^{6}$ A. Nagy, ${ }^{3}$ M.F.A. Nave, ${ }^{41}$ G.A. Navratil, ${ }^{9}$ R. Nazikian, ${ }^{3}$ A. Nerem, ${ }^{1}$ W.M. Nevins, ${ }^{2}$ N. Ohyabu, ${ }^{42}$ T. Oikawa, ${ }^{33}$ M. Okabayashi, ${ }^{3}$ R.A. Olstad, ${ }^{1}$ T.H. Osborne,${ }^{1}$ L.W. Owens, ${ }^{6}$ C.T. Parker, ${ }^{1}$ P.B. Parks, ${ }^{1}$ C.J. Pawley, ${ }^{1}$ L.D. Pearlstein, ${ }^{2}$ J.J. Peavy, ${ }^{1}$ W.A. Peebles, ${ }^{19}$ B.G. Penaflor, ${ }^{1}$ Q. Peng, ${ }^{1}$ F.W. Perkins ${ }^{3}$ P.I. Petersen, ${ }^{1}$ T.W. Petrie,${ }^{1}$ C.C. Petty, ${ }^{1}$ N.Q. Pham,${ }^{1}$ J.C. Phillips, ${ }^{1}$ S.D. Pinches,${ }^{26}$ A. Yu. Pigarov, ${ }^{4}$ D.A. Piglowski, ${ }^{1}$ R.I. Pinsker, ${ }^{1}$ A. Pletzer, ${ }^{3}$ P.A. Politzer, ${ }^{1}$ D. Ponce,${ }^{1}$ M. Porkolab,${ }^{11}$ G.D. Porter, ${ }^{2}$ R. Prater, ${ }^{1}$ D.G. Pretty, ${ }^{28}$ L.E. Randerson, ${ }^{3}$ D.A. Rasmussen, ${ }^{6}$ H. Reimerdes, ${ }^{9}$ E.E. Reis, Jr., ${ }^{1}$ M.E. Rensink, ${ }^{2}$ T.L. Rhodes, ${ }^{19}$ T.D. Rognlien, ${ }^{2}$ M.N. Rosenbluth, ${ }^{1}$ D.W. Ross, ${ }^{5}$ J.C. Rost, ${ }^{11}$ D.L. Rudakov, ${ }^{4}$ E. Ruskov, ${ }^{30}$ F. Ryter, ${ }^{26}$ G. Saibene, ${ }^{26}$ O. Sauter, ${ }^{43}$ R.I. Savercool, ${ }^{1}$ M.J. Schaffer, ${ }^{1}$ D.P. Schissel, ${ }^{1}$ D.J. Schlossberg, ${ }^{15}$ D.D. Schnack, ${ }^{44}$ E. Schuster, ${ }^{36}$ J.T. Scoville, ${ }^{1}$ D. Sellers, ${ }^{1}$ Y. Semenets, ${ }^{14}$ M.W. Shafer ${ }^{15}$ K.C. Shoolbred, ${ }^{1}$ A.C.C. Sips, ${ }^{26}$ A.P. Smirnov, ${ }^{45}$ P.B. Snyder, ${ }^{1}$ E.R. Solano, ${ }^{46}$ W.M. Solomon, ${ }^{3}$ H.E. St John, ${ }^{1}$ W.M. Stacey, ${ }^{40}$ G.M. Staebler, ${ }^{1}$ R.D. Stambaugh, ${ }^{1}$ P.C. Stangeby, ${ }^{18}$ E.J. Strait, ${ }^{1}$ D. Szymanski, ${ }^{1}$ H. Takahashi, ${ }^{3}$ M. Takechi, ${ }^{33}$ P.L. Taylor, ${ }^{1}$ T.S. Taylor, ${ }^{1}$ R.J. Temkin, ${ }^{11}$ T.B. Terpstra, ${ }^{1}$ D.M. Thomas, ${ }^{1}$ P.R. Thomas, ${ }^{8}$ J.F. Tooker, ${ }^{1}$ A.D. Turnbull, ${ }^{1}$ G.R. Tynan, ${ }^{4}$ M.A. Ulrickson, ${ }^{7}$ M.A. VanZeeland, ${ }^{34}$ S. Visser, ${ }^{1}$ M.R. Wade, ${ }^{6}$ F.L. Waelbroeck, ${ }^{5}$ R. Wagner, ${ }^{4}$ M.L. Walker, ${ }^{1}$ R.E. Waltz, ${ }^{1}$ W.R. Wampler, ${ }^{7}$ B. Wan, ${ }^{47}$ G. Wang, ${ }^{19}$ J.G. Watkins, ${ }^{7}$ G.W. Watson, ${ }^{30}$ A.S. Welander, ${ }^{1}$ J.C. Wesley, ${ }^{1}$ W.P. West, ${ }^{1}$ J. Whaley, ${ }^{7}$ D.G. Whyte,${ }^{15}$ H.R. Wilson, ${ }^{13}$ N.S. Wolf, ${ }^{2}$ C.P.C. Wong, ${ }^{1}$ K.-L. Wong, ${ }^{3}$ S.K. Wong, ${ }^{1}$ X.Q. Xu, ${ }^{2}$ H.H. Yip, ${ }^{1}$ L. Zeng, ${ }^{19}$ C. Zhang, ${ }^{47}$ D. Zhou ${ }^{47}$

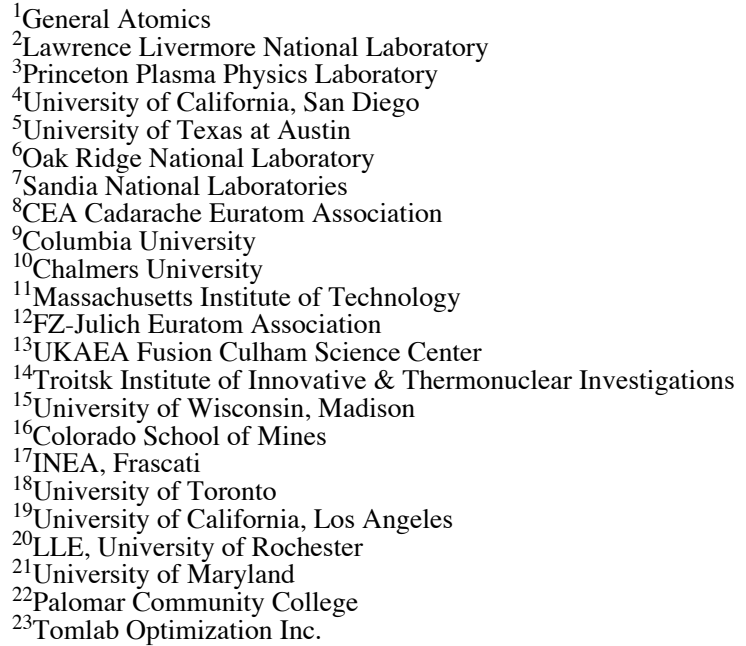

${ }^{1}$ General Atomics

${ }^{2}$ Lawrence Livermore National Laboratory

Princeton Plasma Physics Laboratory

University of California, San Diego

${ }^{7}$ Sandia National Laboratories

${ }^{8}$ CEA Cadarache Euratom Association

Columbia University

${ }^{11}$ Massachusetts Institute of Technology

${ }^{12}$ FZ-Julich Euratom Association

${ }^{13}$ UKAEA Fusion Culham Science Center

${ }^{14}$ Troitsk Institute of Innovative \& Thermonuclear Investigations

sin, Madison

${ }^{19}$ University of California, Los Angeles

LLE, University of Rochester

22 Palomar Community Colleg

${ }^{23}$ Tomlab Optimization Inc.

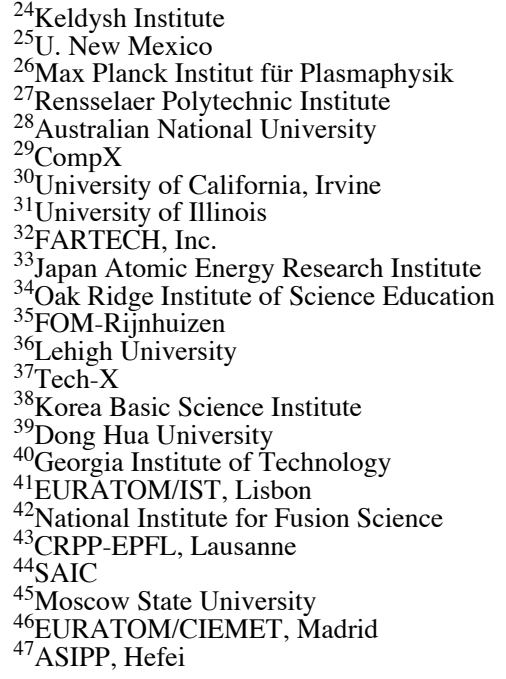

${ }^{24}$ Keldysh Institute

${ }^{26}$ Max Planck Institut für Plasmaphysik

${ }^{27}$ Rensselaer Polytechnic Institute

Australian National University

${ }^{30}$ University of California, Irvine

Research Institute

${ }^{35}$ FOM-Rijnhuizen

${ }^{37}$ Tech-X

${ }^{38}$ Korea Basic Science Institute

${ }^{39}$ Dong Hua University

(n)

${ }^{44} \mathrm{SAIC}$

${ }^{46}$ EURATOM/CIEMET, Madrid

ASIPP, Hefei 